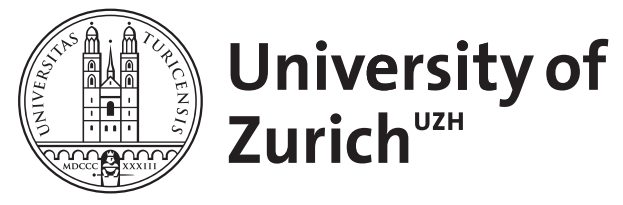

\title{
A spanning tree model for Khovanov homology
}

Wehrli, S

\begin{abstract}
We show that the Khovanov complex of a connected link diagram D retracts to a subcomplex whose generators are in 2:1 correspondence with the spanning trees of the "black graph" of D. Using this result, we give a new proof of Lee's theorem on the support of Khovanov homology of alternating knots.
\end{abstract}

DOI: https://doi.org/10.1142/S0218216508006762

Posted at the Zurich Open Repository and Archive, University of Zurich ZORA URL: https://doi.org/10.5167/uzh-21462

Journal Article

Accepted Version

Originally published at:

Wehrli, S (2008). A spanning tree model for Khovanov homology. Journal of Knot Theory and Its Ramifications (JKTR), 17(12):1561-1574.

DOI: https://doi.org/10.1142/S0218216508006762 


\title{
A spanning tree model for Khovanov homology
}

\author{
Stephan Wehrli, University of Basel, August 2004
}

\begin{abstract}
We use a spanning tree model to prove a result of E. S. Lee on the support of Khovanov homology of alternating knots.
\end{abstract}

\section{Introduction}

In [Th], M. Thistlethwaite observed that the Kauffman bracket of a knot diagram is related to the Tutte polynomial of the "black graph" of the knot diagram. In particular, the Kauffman bracket may be expanded as a sum over terms corresponding to spanning trees of the "black graph" of the knot diagram. More than a year ago, the author constructed an analogue of this expansion for Khovanov homology. The idea to use a spanning tree model for calculating Khovanov homology was considered by other people independently. ${ }^{1}$ In this paper, we use the spanning tree model for Khovanov homology to give a new proof of a theorem due to E. S. Lee [Lee-1] on the support of the Khovanov homology of alternating knots. The paper is organized as follows: in Section 1, we briefly review Thistlethwaite's construction, however without making any reference to the Tutte polynomial. In Section 2, we describe how the spanning tree model for the Kauffman bracket leads to a spanning tree model for Khovanov homology. We give a short proof of a theorem on the behavior of Khovanov homology under Hopf link addition. This theorem was first proved by M. Asaeda and J. Przytycki in $[\mathrm{AP}]$. In Section 3, we use the results of Section 2 to prove Lee's theorem [Lee-1]. In Section 4, we discuss a spanning tree model for a homology theory defined in [Lee-2].

\section{Spanning tree model for the Kauffman bracket}

Unless otherwise stated, link diagrams are assumed to be unoriented and equipped with a numbering of the crossings. Let $D$ be a link diagram and let $\times$ be a crossing of $D$. We may smoothen $\times$ by replacing it either by ) ( or

\footnotetext{
${ }^{1}$ I. Kofman independently brought up the idea of a spanning tree model for Khovanov homology at the "Knots in Poland" conference in July 2003, together with O. Viro, M. Polyak, A. Shumakovitch and L. Kauffman. I. Kofman [Ko] gave a talk with the title "Spanning trees and Khovanov homology" at the "Knots in Washington XVIII" conference in May 2004 (which the author did not attend). The topic of this paper is very similar to that of I. Kofman's talk, yet all results were obtained independently. A paper on the subject by I. Kofman, A. Champanerkar and O. Viro is in preparation.
} 
by $\asymp$. As in $[\mathrm{BN}]$, we name ) (the 0-smoothing and $\asymp$ the 1-smoothing of ×. For a diagram $D^{\prime}$ obtained from $D$ by smoothening some of the crossings of $D$, we define $<D \mid D^{\prime}>\in \mathbb{Z}\left[q, q^{-1}\right]$ by $<D \mid D^{\prime}>:=(-q)^{r\left(D, D^{\prime}\right)}$, where $r\left(D, D^{\prime}\right)$ denotes the number of 1 -smoothings in $D^{\prime}$. A diagram obtained from $D$ by smoothening all of the crossings of $D$ is called a Kauffman state of $D$. Let $\mathcal{K}(D)$ denote the set of all Kauffman states of $D$ and $\mathcal{K}_{k}(D)$ the set of all Kauffman states of $D$ consisting of exactly $k$ disjoint circles. Given a link diagram $D$, M. Khovanov [Kh-1] assigns a Laurent polynomial $<D>\in \mathbb{Z}\left[q, q^{-1}\right]$ by the rules

$$
\begin{gathered}
<\times>=<)(>-q<\asymp>, \\
<O^{k}>=\left(q+q^{-1}\right)^{k} .^{2}
\end{gathered}
$$

$<D>$ is a scaled version of the Kauffman bracket [Ka-2]. It is invariant under Reidemeister moves, up to multiplication with a unit of $\mathbb{Z}\left[q, q^{-1}\right]$. There is an explicit formula for the Kauffman bracket:

$$
<D>=\sum_{D^{\prime} \in \mathcal{K}(D)}<D \mid D^{\prime}><D^{\prime}>
$$

To obtain (3) from (1), we may proceed as follows: First, we apply relation (1) to crossing number 1 to express $\langle D\rangle$ as the sum of the two terms on the right-hand side of (1). Next, we apply relation (1) to crossing number 2 to express each of these two terms as a sum of two other terms, and so on. This procedure is visualized in the binary tree in Figure 1:

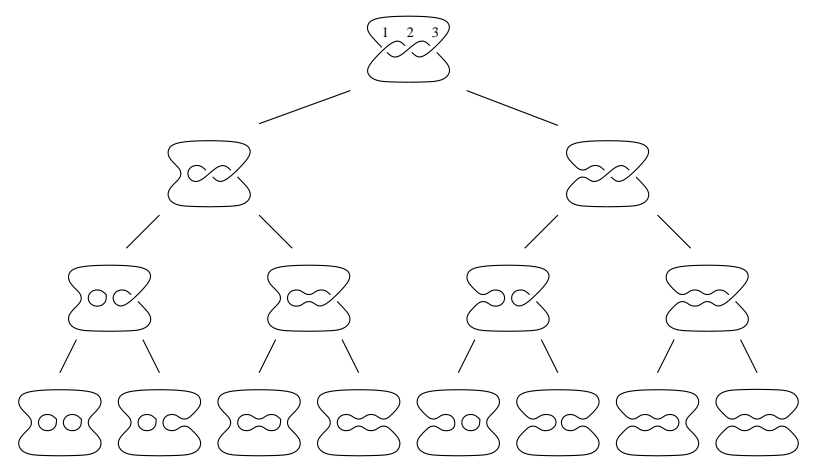

Figure 1: Binary tree used to deduce (3) from (1).

The diagrams sitting at the leaves of the tree are the Kauffman states of $D$, whence (3) follows.

\footnotetext{
${ }^{2}$ Х, $)(, \asymp$ denote any three link diagrams which agree except in a small disk where they look like $\times),\left(, \asymp\right.$, respectively. $O^{k}$ denotes a diagram without crossings which consists of $k$ disjoint circles.
} 
Unfortunately, the complexity of (3) grows exponentially in the complexity of $D$. In case $D$ is connected, we get a more efficient formula by modifying the above procedure as follows: each time before applying relation (1), we check the connectivity of the diagrams on the right-hand side. We rewrite the term on the left-hand side as the sum of the terms on the right-hand side only if both diagrams on the right-hand side are connected. The modified procedure is visualized in the binary tree in Figure 2:

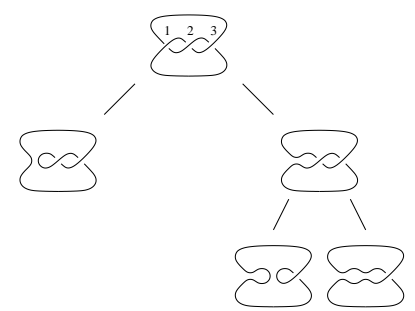

Figure 2: Binary tree used to deduce (4) from (1).

We immediately obtain:

$$
<D>=\sum_{D^{\prime} \in \mathcal{T}(D)}<D \mid D^{\prime}><D^{\prime}>,
$$

where $\mathcal{T}(D)$ denotes the set of all diagrams sitting at the leaves of the tree in Figure 2. (Note that $\mathcal{T}(D)$ may depend on the numbering of the crossings of $D$.) Let $D^{\prime}$ be an element of $\mathcal{T}(D)$. By construction $D^{\prime}$ is connected and every crossing of $D^{\prime}$ is splitting (i.e. connects two otherwise disconnected parts of $D^{\prime}$ ). A diagram with these properties will be called R1-trivial because it can be trivialized using Reidemeister move 1 only.

It is easy to see that the Kauffman bracket behaves as follows under Reidemeister move 1:

$$
\left.\left.<\ngtr>=q^{-1}<\right)>, \quad<\succ>=-q^{2}<\right)>\text {. }
$$

From (5) we get an explicit formula for the Kauffman bracket of an R1-trivial diagram: Let $D^{\prime}$ be an R1-trivial diagram and assume that an orientation of $D^{\prime}$ is given. Let $x\left(D^{\prime}\right)$ denote the number of negative $(\chi)$ crossings of $D^{\prime}$ and $y\left(D^{\prime}\right)$ the number of positive ( $\left.\times\right)$ crossings of $D^{\prime}$. Then

$$
<D^{\prime}>=(-1)^{x\left(D^{\prime}\right)} q^{2 x\left(D^{\prime}\right)-y\left(D^{\prime}\right)}\left(q+q^{-1}\right) .
$$

Since $\mathcal{K}_{1}(D)=\bigsqcup_{D^{\prime} \in \mathcal{T}(D)} \mathcal{K}_{1}\left(D^{\prime}\right)$ and since $\# \mathcal{K}_{1}\left(D^{\prime}\right)=1$ for $D^{\prime} \mathrm{R} 1$ trivial, the elements of $\mathcal{K}_{1}(D)$ correspond bijectively to the elements of $\mathcal{T}(D)$. Indeed, for $S \in \mathcal{K}_{1}(D)$ let $D_{S}$ be the unique element of $\mathcal{T}(D)$ having $S$ among its Kauffman states. We may rewrite (4) as

$$
<D>=\sum_{S \in \mathcal{K}_{1}(D)}<D \mid D_{S}><D_{S}>.
$$


Assume that the regions of the knot projection underlying the knot diagram $D$ are colored black and white in a chessboard pattern, i.e. such that any two regions which share an edge have opposite colors and such that the unbounded region is colored white. (There is exactly one such coloring. It is obtained by coloring the bounded and the unbounded region of any $S \in \mathcal{K}_{1}(D)$ black and white, respectively.) A smoothing of a crossing of $D$ will be called a black smoothing or a white smoothing depending on whether it connects two black regions or two white regions of $D$. The black graph of $D$ is the graph whose vertices correspond to the black regions of $D$ and which has an edge connecting two vertices for each crossing where the corresponding black regions touch. There is a bijection between spanning trees of the black graph of $D$ and elements of $\mathcal{K}_{1}(D)$ defined as follows: to a spanning tree assign an element of $\mathcal{K}_{1}(D)$ by choosing the black smoothing for precisely those crossings of $D$ which correspond to edges of the spanning tree. We call (7) a spanning tree model for the Kauffman bracket. ${ }^{3}$

Since the number of edges in a tree is one less than the number of vertices, the number of black smoothings in an element of $\mathcal{K}_{1}(D)$ is one less than the number of black regions of $D$. In particular, the number of black smoothings is the same for all $S \in \mathcal{K}_{1}(D)$. This fact will be important, so let us give another proof of it: by the Clock Theorem [Ka-1], any two elements of $\mathcal{K}_{1}(D)$ are related by a finite sequence of state transpositions (see Figure 3 ) and state transpositions do not change the number of black smoothings.
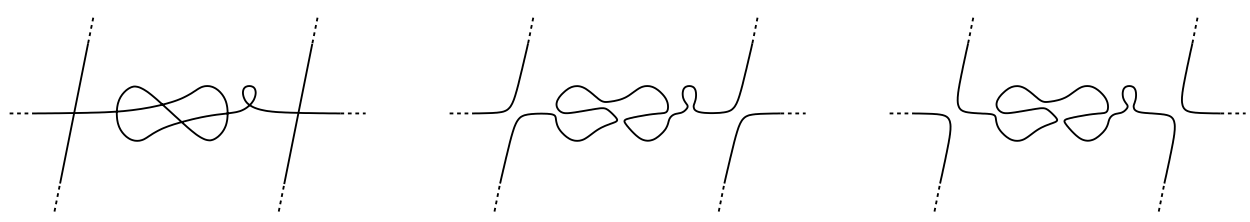

Figure 3: A knot projection and two smoothings related by a state transposition.

The fact that the number of black smoothings in $S \in \mathcal{K}_{1}(D)$ is independent of $S$ may also be shown by induction on the number of crossings of $D$.

\section{Spanning tree model for Khovanov homology}

In [Kh-1], M. Khovanov assigns to a link diagram $D$ a bigraded complex $\overline{\mathcal{C}}(D)$ with differential $d$ of bidegree $(1,0)$. Let $\overline{\mathcal{H}}(D)$ denote the homology of $\overline{\mathcal{C}}(D)$. We call $\overline{\mathcal{C}}(D)$ the Khovanov complex of $D$ and $\overline{\mathcal{H}}(D)$ the Khovanov homology. The Khovanov complex is a link invariant when considered up to shifts of the gradings and up to a chain equivalence which preserves the

\footnotetext{
${ }^{3}$ Although our approach is different, formula (7) is essentially the same as the formula used to define $\Gamma_{G}$ in [Th]. Note that both formulae depend on a numbering of the crossings. To go from (7) to the fomula in [Th], one has to reverse the order of the crossings.
} 
secondary grading (and also the primary grading, but this is already contained in the definition of a chain equivalence). M. Khovanov's construction may be viewed as a categorification of the Kauffman bracket. Indeed, the Kauffman bracket is the "graded Euler characteristic" of the Khovanov complex (see [Kh-1]). In this section, we construct an analogue of (7) for the Khovanov complex. We use the following notations and conventions: all modules and complexes are assumed to be bigraded. Isomorphisms between bigraded objects are assumed to preserve the gradings. Direct sums are assumed to be compatible with the gradings. If $M$ is a bigraded object, we denote by $M^{i, j}$ its homogeneous component of bidegree $(i, j)$. For $m, n \in \mathbb{Z},[m]$ and $\{n\}$ denote the operators on bigraded objects defined by $(M[m]\{n\})^{i, j}:=M^{i-m, j-n}$. Unless otherwise stated, we work with coefficients in $\mathbb{Z}$.

Instead of giving the precise definition of $\overline{\mathcal{C}}(D)$, we list the properties of $\overline{\mathcal{C}}(D)$ which are relevant to our discussion. On the level of modules,

$$
\overline{\mathcal{C}}(D)=\bigoplus_{D^{\prime} \in \mathcal{K}(D)} \overline{\mathcal{C}}\left(D^{\prime}\right)\left[r\left(D, D^{\prime}\right)\right]\left\{r\left(D, D^{\prime}\right)\right\}
$$

In particular, $\overline{\mathcal{C}}($ メ $)$ as a module is canonically isomorphic to $\overline{\mathcal{C}}()() \oplus \overline{\mathcal{C}}(\asymp)[1]\{1\}$. On the level of complexes, the modification $)(\rightsquigarrow \asymp$ induces a chain transformation

$$
w: \overline{\mathcal{C}}()() \longrightarrow \overline{\mathcal{C}}(\asymp)\{1\}
$$

which preserves the secondary grading and such that $\overline{\mathcal{C}}(\times)$ is canonically isomorphic to the mapping cone ${ }^{4}$ of $w$. The Khovanov complex of a diagram without crossings has trivial differential. As a module, it is given by

$$
\overline{\mathcal{C}}\left(O^{k}\right)=\mathcal{A}^{\otimes k}
$$

where $\mathcal{A}$ is the bigraded module defined by $\mathcal{A}^{0,-1}=\mathcal{A}^{0,1}=\mathbb{Z}$ and $\mathcal{A}^{i, j}=0$ for $(i, j) \neq(0, \pm 1)$. The gradings are additive under tensor multiplication. By [Kh-1, Section 5], the behavior of the Khovanov complex under Reidemeister move 1 is as follows:

$$
\left.\overline{\mathcal{C}}(\supset) \cong \overline{\mathcal{C}}())\{-1\} \oplus B_{1}, \quad \overline{\mathcal{C}}(\succ) \cong \overline{\mathcal{C}}()\right)[1]\{2\} \oplus B_{2}
$$

for contractible ${ }^{5}$ complexes $B_{1}, B_{2}$.

\footnotetext{
${ }^{4}$ Let $w$ be a chain transformation from a complex $C_{0}$ with differential $d_{0}$ to a complex $C_{1}$ with differential $d_{1}$. The mapping cone of $w$ is the complex $C$ with differential $d$ defined as follows: As a module, $C=C_{0} \oplus C_{1}[1]$. The restriction of $d$ to $C_{0}$ is $d_{0}+w$. The restriction of $d$ to $C_{1}[1]$ is $-d_{1}$.

${ }^{5}$ A complex $B$ with differential $d$ of bidegree $(1,0)$ is called contractible if it is chain equivalent to the trivial complex, i.e. if there exists a graded module endomorphism $p$ of $B$ of bidegree $(-1,0)$ such that $p \circ d+d \circ p=i d_{B}$. Note that a contractible complex has trivial homology.
} 
We are now ready to discuss how (7) transfers to the Khovanov complex. Consider the Khovanov complex of a diagram sitting at an internal node in the binary tree of Figure 2. It is canonically isomorphic to the mapping cone of a chain transformation between the Khovanov complexes of the two diagrams sitting right below the given diagram in the binary tree. As a module, it is equal to the direct sum of the modules underlying these two complexes. (Actually, there are also some shifts of the gradings.) Hence on the level of modules

$$
\overline{\mathcal{C}}(D)=\bigoplus_{S \in \mathcal{K}_{1}(D)} \overline{\mathcal{C}}\left(D_{S}\right)\left[r\left(D, D_{S}\right)\right]\left\{r\left(D, D_{S}\right)\right\}
$$

By (11), the complexes $\overline{\mathcal{C}}\left(D_{S}\right)$ admit decompositions

$$
\overline{\mathcal{C}}\left(D_{S}\right) \cong \mathcal{A}\left[x\left(D_{S}\right)\right]\left\{2 x\left(D_{S}\right)-y\left(D_{S}\right)\right\} \oplus B\left(D_{S}\right),
$$

for contractible complexes $B\left(D_{S}\right)$. To obtain a decomposition of the complex $\overline{\mathcal{C}}(D)$, we need the following lemma which asserts that forming the direct sum with a contractible complex "commutes", up to isomorphism, with the mapping cone construction:

Lemma 2.1 Let $C_{0}$ and $C_{1}$ be complexes with $C_{i}=A_{i} \oplus B_{i}$ for complexes $A_{i}, B_{i}$ with $B_{i}$ contractible. Let $w: C_{0} \rightarrow C_{1}$ be a grading-preserving chain transformation and let $w_{A A}: A_{0} \rightarrow A_{1}$ denote $w$ composed with the obvious projection and inclusion. Let $A, B$ and $C$ be the mapping cone of $w_{A A}$, the (contractible) complex $B_{0} \oplus B_{1}[1]$ and the mapping cone of $w$, respectively. Then $C \cong A \oplus B$.

Proof. Define $w_{A B}: B_{0} \rightarrow A_{1}, w_{B A}: A_{0} \rightarrow B_{1}$ and $w_{B B}: B_{0} \rightarrow B_{1}$ in the same way as $w_{A A}$. Since $B_{i}$ is contractible, there exists $p_{i}$ with $p_{i} \circ d_{i}+d_{i} \circ p_{i}=$ $i d_{B_{i}}$. Writing elements of both $C$ and $A \oplus B$ in the form $\left(a_{0}, b_{0}, a_{1}, b_{1}\right)$, $a_{i} \in A_{i}, b_{i} \in B_{i}$, we define $f: C \rightarrow A \oplus B$ by $f\left(a_{0}, b_{0}, a_{1}, b_{1}\right):=\left(a_{0}, b_{0}, a_{1}-\right.$ $\left.\left(w_{A B} \circ p_{0}\right) b, b_{1}-\left(w_{B B} \circ p_{0}\right) b-\left(p_{1} \circ w_{B A}\right) a\right)$. Direct calculation shows that $f$ is an isomorphism of complexes.

In view of $(12),(13)$ and Lemma 2.1 , it is easy to see that the complex $\overline{\mathcal{C}}(D)$ is isomorphic to a direct sum of two complexes, $A$ and $B$ say, where $B$ is the direct sum of the $B\left(D_{S}\right)$ (up to shifts of the gradings) and $A$ as a module is given by

$$
A=\bigoplus_{S \in \mathcal{K}_{1}(D)} \mathcal{A}\left[x\left(D_{S}\right)\right]\left\{2 x\left(D_{S}\right)-y\left(D_{S}\right)\right\}\left[r\left(D, D_{S}\right)\right]\left\{r\left(D, D_{S}\right)\right\} .
$$

Writing $w\left(D_{S}\right)$ for $x\left(D_{S}\right)-y\left(D_{S}\right)$ and observing that $r\left(D, D_{S}\right)=r(D, S)-$ $r\left(D_{S}, S\right)=r(D, S)-y\left(D_{S}\right)$, we obtain the following theorem: 
Theorem 2.2 Let $D$ be a connected link diagram. Then there is a decomposition $\overline{\mathcal{C}}(D) \cong A \oplus B$, where $B$ is contractible and $A$ as a module is given by

$$
A=\bigoplus_{S \in \mathcal{K}_{1}(D)} \mathcal{A}\left[w\left(D_{S}\right)\right]\left\{2 w\left(D_{S}\right)\right\}[r(D, S)]\{r(D, S)\} .
$$

We call the decomposition $\overline{\mathcal{C}}(D) \cong A \oplus B$ together with the above formula a spanning tree model for Khovanov homology.

Although the above constructions are completely explicit, it may be difficult to compute the differential of $A$ in practice. However, we have the estimates $\operatorname{dim}_{\mathbb{Q}}(\overline{\mathcal{H}}(D) \otimes \mathbb{Q}) \leq \operatorname{rank}(A)$ and $\operatorname{dim}_{\mathbb{Q}}\left(\overline{\mathcal{H}}(D)^{i, j} \otimes \mathbb{Q}\right) \leq \operatorname{rank}\left(A^{i, j}\right)$, which together with (15) imply the following theorem:

Theorem 2.3 Let $D$ be a connected link diagram. Then

$$
\operatorname{dim}_{\mathbb{Q}}(\overline{\mathcal{H}}(D) \otimes \mathbb{Q}) \leq 2\left(\# \mathcal{K}_{1}(D)\right) .
$$

Moreover, the dimension of $\overline{\mathcal{H}}(D)^{i, j} \otimes \mathbb{Q}$ is bounded from above by the number of $S \in \mathcal{K}_{1}(D)$ with $w\left(D_{S}\right)+r(D, S)=i$ and $r(D, S)=2 i-j \pm 1$.

Theorem 2.3 shows that the dimension of $\overline{\mathcal{H}}(D) \otimes \mathbb{Q}$ tends to be much smaller than the rank of $\overline{\mathcal{C}}(D)$, a fact observed experimentally by D. Bar-Natan [BN].

Remark. In $[\mathrm{Kh}-2], H^{1}:=\mathcal{A}\{-1\}$ is endowed with the structure of a $\mathbb{Z}_{--}$ graded $^{6}$ commutative ring. In particular, $\mathcal{A}$ is an $H^{1}$-module and $\mathcal{A}^{0,-1} \subset \mathcal{A}$ an $H^{1}$-submodule. When a distinguished point on the knot projection (which is not a double point) is chosen, $\overline{\mathcal{C}}(D)$ becomes a complex of $H^{1}$ modules. The isomorphisms in (11) are isomorphisms of $H^{1}$-modules, provided Reidemeister move 1 is performed away from the distinguished point. Hence we may assume that the decomposition of $\overline{\mathcal{C}}(D)$ given in Theorem 2.2 is compatible with the $H^{1}$-module structure. By (15), $A$ is generated as an $H^{1}$-module by elements corresponding to elements of $\mathcal{K}_{1}(D)$, i.e. to spanning trees of the black graph of $D$. Similarly, the reduced Khovanov complexes $\overline{\mathcal{C}}(D) \otimes_{H^{1}} \mathcal{A}^{0,-1}$ and $\overline{\mathcal{C}}(D) \otimes_{H^{1}}\left(\mathcal{A} / \mathcal{A}^{0,-1}\right)$ are chain equivalent to complexes which are generated as $\mathbb{Z}$-modules by elements corresponding to spanning trees of the black graph of $D$. It is interesting to compare this with knot Floer homology (see $[\mathrm{OSz}]$ ).

Remark. By [Kh-2], there is an isomorphism $\overline{\mathcal{C}}\left(D_{1} \# D_{2}\right) \cong \overline{\mathcal{C}}\left(D_{1}\right) \otimes_{H^{1}}$ $\overline{\mathcal{C}}\left(D_{2}\right)\{-1\}$. (Here, the $H^{1}$-module structures on $\overline{\mathcal{C}}\left(D_{1}\right)$ and $\overline{\mathcal{C}}\left(D_{2}\right)$ are defined as in the previous remark, by choosing distinguished points on $D_{1}$ and $D_{2}$ close to the connected sum point.) Let $D_{2}$ be a standard diagram of the Hopf link. By Theorem 2.2, $\overline{\mathcal{C}}\left(D_{2}\right) \cong A \oplus B$ where $B$ is contractible and $A$ as an $H^{1}$-module is equal to $\mathcal{A}[-1]\{-2\} \oplus \mathcal{A}[1]\{2\}$. The differential of $A$ has

\footnotetext{
${ }^{6}$ Our grading on $H^{1}$ is opposite to the grading used in [Kh-2].
} 
bidegree $(1,0)$, whence it must be zero. We conclude that $\overline{\mathcal{C}}\left(D_{1} \# D_{2}\right)$ is chain equivalent to $\overline{\mathcal{C}}\left(D_{1}\right)[-1]\{-2\} \oplus \overline{\mathcal{C}}\left(D_{1}\right)[1]\{2\}$. Consequently, $\overline{\mathcal{H}}\left(D_{1} \# D_{2}\right)$ is isomorphic to $\overline{\mathcal{H}}\left(D_{1}\right)[-1]\{-2\} \oplus \overline{\mathcal{H}}\left(D_{1}\right)[1]\{2\}$. The latter was first proved by M. Asaeda and J. Przytycki $[\mathrm{AP}]$, who thus confirmed a conjecture of A. Shumakovitch [Sh-1]. A special case was already considered in [We, Section 4].

\section{Support of Khovanov homology for alternating knots}

The theorems in this section were conjectured by D. Bar-Natan, S. Garoufalidis and M. Khovanov [BN] and proved by E. S. Lee [Lee-1]. We give new proofs using the spanning tree model. For short proofs, see also [AP].

A knot diagram is alternating if one alternately over- and undercrosses other strands as one goes along the knot in that diagram. A knot is called alternating if it possesses an alternating diagram.

Let $D$ be an alternating knot diagram. Then either the black smoothing coincides with the 1-smoothing for all crossings or the white smoothing coincides with the 1-smoothing for all crossings. By Section 1, the numbers of black and white smoothings in $S \in \mathcal{K}_{1}(D)$ are independent of $S$. Therefore $r(D, S)$ is independent of $S \in \mathcal{K}_{1}(D)$. Let $n_{1}(D):=r(D, S)$ for any $S \in$ $\mathcal{K}_{1}(D)$. From Theorem 2.2 we get:

Theorem 3.1 Let $D$ be an alternating knot diagram. Then $\overline{\mathcal{H}}(D)^{i, j}=0$ unless $(i, j)$ lies on one of the two lines $j=2 i-n_{1}(D) \pm 1$. Moreover, $\overline{\mathcal{H}}(D)^{i, j}$ is torsion free unless $j=2 i-n_{1}(D)-1$.

The statement about the torsion follows from the fact that the differential of $A$ has bidegree $(1,0)$. We also obtain:

Theorem 3.2 Let $D$ be an alternating knot diagram. Let $i_{-}$and $i_{+}$be the smallest and largest primary degree in which $\overline{\mathcal{H}}(D)$ is non-zero. Then $\overline{\mathcal{H}}(D)^{i_{-}, j_{-}}$and $\overline{\mathcal{H}}(D)^{i_{+}, j_{+}}$are non-zero for $\left(i_{-}, j_{-}\right)$on the lower line and $\left(i_{+}, j_{+}\right)$on the upper line (i.e. $j_{-}=2 i_{-}-n_{1}(D)-1$ and $j_{+}=2 i_{+}-$ $\left.n_{1}(D)+1\right)$.

The following theorem corresponds to parts (i) and (iii) of Theorem 1 of [Th]. Our proof will be related to the proofs given in [Th]. For a different proof of a similar statement, see [Kh-1, Section 7.7].

Theorem 3.3 Let $D$ be an alternating knot diagram. Assume that no crossing of $D$ is splitting. Then $\overline{\mathcal{H}}(D)^{i_{-}, j_{-}}=\overline{\mathcal{H}}(D)^{i_{+}, j_{+}}=\mathbb{Z}$ and $i_{-}=0$ and $i_{+}$ is equal to the number of crossings of $D$.

Theorem 3.3 is a consequence of Theorem 2.2 and the following lemma, in which $n_{0}(D)$ denotes the number of 0 -smoothings in any $S \in \mathcal{K}_{1}(D)$ : 
Lemma 3.4 Let $D$ be an alternating knot diagram. Assume that no crossing of $D$ is splitting. For any $S \in \mathcal{K}_{1}(D)$ there is a numbering of the crossings of $D$ such that $w\left(D_{S}\right)=-n_{1}(D)$ and $w\left(D_{S^{\prime}}\right)>-n_{1}(D)$ for $S^{\prime} \neq S$. Likewise, there is a numbering of the crossings of $D$ such that $w\left(D_{S}\right)=n_{0}(D)$ and $w\left(D_{S^{\prime}}\right)<n_{0}(D)$ for $S^{\prime} \neq S$.

Proof of the lemma. Let $S \in \mathcal{K}_{1}(D)$. Let us number the crossings of $D$ in such a way that the crossings which are 0-smoothings in $S$ precede those which are 1-smoothings in $S$. Since either the black smoothing coincides with the 1-smoothing for all crossings or the white smoothing coincides with the 1-smoothing for all crossings and since no crossing of $D$ is splitting, the construction of $D_{S}$ implies that the crossings of $D$ which are not smoothened in $D_{S}$ are precisely those which are 1-smoothings in $S$. Moreover, a look at local orientations shows that these crossings have to be positive with respect to any orientation of $D_{S}$, for otherwise $S$ could not be connected. So $w\left(D_{S}\right)=-y\left(D_{S}\right)=-n_{1}(D)$. Now let us consider $S^{\prime} \in \mathcal{K}_{1}(D)$ with $S^{\prime} \neq S$. The first crossing of $D$ where $S$ and $S^{\prime}$ differ has to be a 0 -smoothing in $S$ and a 1-smoothing in $S^{\prime}$. Moreover, it must be smoothened in $D_{S^{\prime}}$ because it is smoothened in $D_{S}$. We conclude $y\left(D_{S^{\prime}}\right)<y\left(D_{S}\right)$, whence $w\left(D_{S^{\prime}}\right)>-y\left(D_{S}\right)=-n_{1}(D)$. Analogously, if we number the crossings of $D$ in such a way that the crossings which are 1-smoothings in $S$ precede those which are 0-smoothings in $S$, we have $w\left(D_{S}\right)=n_{0}(D)$ and $w\left(D_{S^{\prime}}\right)<n_{0}(D)$ for $S^{\prime} \neq S$.

Note that the difference $i_{+}(D)-i_{-}(D)$ is a knot invariant. By (8), it cannot exceed the number of crossings of $D$. From Theorem 3.3 we get the following corollary which coincides with Corollary 1 of [Th]:

Corollary 3.5 If a knot possesses an alternating diagram with $m$ crossings, all of which are non-splitting, then the knot does not admit a diagram with fewer than $m$ crossings.

\section{Spanning tree model and Lee's differential}

In [Lee-2], E. S. Lee defined a differential $\Phi$ of bidegree $(1,4)$ on $\overline{\mathcal{C}}(D)$ which anticommutes with the differential $d$. It follows that $(d+\Phi)^{2}=0$, whence $d^{\prime}:=d+\Phi$ may be considered as a differential on $\overline{\mathcal{C}}(D)$. Note that $d^{\prime}$ does not decrease the secondary degree. Otherwise stated, $d^{\prime}$ respects the filtration defined as follows: an element of $\overline{\mathcal{C}}(D)$ has filtration at least $j$ if and only if it is a sum of homogeneous elements of secondary degree at least $j$. Let $\overline{\mathcal{C}}^{\prime}(D)$ denote the complex $\overline{\mathcal{C}}(D)$ with differential $d^{\prime}$ instead of $d$.

Theorem 4.1 The complex $\overline{\mathcal{C}}^{\prime}(D)$ is invariant under Reidemeister moves, up to shift of the primary grading and up to filtered chain equivalence. In 
particular, there are decompositions

$$
\left.\left.\overline{\mathcal{C}}^{\prime}(\diamond) \cong \overline{\mathcal{C}}^{\prime}()\right)\{-1\} \oplus B_{1}^{\prime}, \quad \overline{\mathcal{C}}^{\prime}(\supset) \cong \overline{\mathcal{C}}^{\prime}()\right)[1]\{2\} \oplus B_{2}^{\prime}
$$

for contractible filtered complexes $B_{1}^{\prime}$ and $B_{2}^{\prime}$. These decompositions respect the filtration (meaning that the associated inclusion and projection maps respect the filtration).

To prove Theorem 4.1, we may adopt the proofs of the corresponding statements for the Khovanov complex given in [Kh-1, Section 5]. Let us explain this in more detail. Let $\mathcal{M}$ denote the category which has closed 1-manifolds as objects and cobordisms as morphisms (and disjoint union as tensor product). The definition of the Khovanov complex given in [Kh-1] involves a monoidal functor $\mathcal{F}$ from $\mathcal{M}$ to the category which has graded $\mathbb{Z}$-modules as objects and graded $\mathbb{Z}$-module homomorphisms as morphisms. On objects, $\mathcal{F}$ is given by $\mathcal{F}\left(O^{k}\right)=\mathcal{A}^{\otimes k} . \mathcal{F}$ satisfies $\mathcal{F}\left(S_{0}^{0}\right)=0$ and

$$
\mathcal{A} \otimes \mathcal{A}=\mathcal{F}\left(S_{1}^{1} \sqcup S_{0}^{1}\right) \mathcal{A} \oplus \mathcal{F}\left(S_{1}^{2}\right) \mathcal{A},
$$

where $S_{k}^{l}$ denotes the cobordism from $O^{k}$ to $O^{l}$ which is a 2 -sphere with $k+l$ disks removed. Note that the inclusion and projection maps associated with decomposition (18) may be described in terms of the functor $\mathcal{F}$. (For example, the projection onto $\mathcal{F}\left(S_{1}^{1} \sqcup S_{0}^{1}\right) \mathcal{A}$ is given by $\mathcal{F}\left(S_{2}^{1}\right) \circ\left(\mathcal{F}\left(S_{1}^{1} \sqcup S_{1}^{1}\right)\right.$ $\left.\mathcal{F}\left(S_{1}^{2}\right) \circ \mathcal{F}\left(S_{1}^{1} \sqcup S_{1}^{0}\right)\right)$. To verify this, use $\mathcal{F}\left(S_{0}^{0}\right)=0$ and the functoriality of $\mathcal{F}$.) In $[\mathrm{Ra}]$, J. Rasmussen remarked that $\overline{\mathcal{C}}^{\prime}(D)$ may be defined by using a monoidal functor $\mathcal{F}^{\prime}$ from $\mathcal{M}$ to the category which has filtered $\mathbb{Z}$-modules as objects and filtered $\mathbb{Z}$-module homomorphisms as morphisms. $\mathcal{F}^{\prime}$ satisfies $\mathcal{F}^{\prime}\left(O^{k}\right)=\mathcal{A}^{\otimes k}$ and $\mathcal{F}^{\prime}\left(S_{0}^{0}\right)=0$. It is straightforward to see that (18) remains satisfied when $\mathcal{F}$ is replaced by $\mathcal{F}^{\prime}$. Now Theorem 4.1 may be established by replacing $\mathcal{F}$ by $\mathcal{F}^{\prime}$ in the proofs of [Kh-1, Section 5]. Note that these proofs rely only on (18) and on the functoriality of $\mathcal{F}$.

From Theorem 4.1 and the definition of $\overline{\mathcal{C}}^{\prime}(D)$ it is clear that the properties of the Khovanov complex used to deduce Theorem 2.2 have analogues for $\overline{\mathcal{C}}^{\prime}(D)$. Therefore, we obtain:

Theorem 4.2 Let $D$ be a connected link diagram. Then $\overline{\mathcal{C}}^{\prime}(D) \cong A \oplus B$ where $B$ is a contractible filtered complex and $A$ as a filtered module is given by (15). The decomposition $\overline{\mathcal{C}}^{\prime}(D) \cong A \oplus B$ respects the filtration.

The remarks at the end of Section 2 remain true for $\overline{\mathcal{C}}^{\prime}(D)$ if the multiplication on $H^{1}=\mathcal{A}\{-1\}$ is replaced by the multiplication induced by $\mathcal{F}^{\prime}\left(S_{2}^{1}\right): \mathcal{A} \otimes \mathcal{A} \rightarrow \mathcal{A}$ and $\mathcal{A}^{0,-1} \subset \mathcal{A}$ is replaced by $\mathbb{Z} a \subset \mathcal{A}$, for $a \in \mathcal{A}$ defined as in [Lee-2]. Let $\overline{\mathcal{H}}^{\prime}(D)$ denote the bigraded module given by $\overline{\mathcal{H}}^{\prime}(D)^{i, j}:=F^{i, j} / F^{i, j-1}$, where $F^{i, j}$ is the submodule of the homology of $\overline{\mathcal{C}}^{\prime}(D)$ consisting of all homology classes which have representatives of primary degree $i$ and filtration at least $j$. The first part of Theorem 3.1 remains 
true if $\overline{\mathcal{H}}(D)$ is replaced by $\overline{\mathcal{H}}^{\prime}(D)$. In general, the statement about the torsion is not true for $\overline{\mathcal{H}}^{\prime}(D)$.

Example. Let $D$ be a standard diagram of the left handed trefoil. Then $\overline{\mathcal{C}}^{\prime}(D) \cong A \oplus B$ for a contractible complex $B$ and $A=\mathcal{A}[0]\{-1\} \oplus \mathcal{A}[2]\{3\} \oplus$ $\mathcal{A}[3]\{5\}$, as a module. The differential of $A$ is zero on $\mathcal{A}[0]\{-1\}$ and maps $1, X \in \mathcal{A}[2]\{3\}$ to $2 X, 2 \cdot 1 \in \mathcal{A}[3]\{5\}$, respectively. Whence $\overline{\mathcal{H}}^{\prime}(D) \cong$ $\mathcal{A}[0]\{-1\} \oplus(\mathcal{A} / 2 \mathcal{A})[3]\{5\}$. In particular, $\overline{\mathcal{H}}^{\prime}(D)^{3,6} \cong \mathbb{Z} / 2 \mathbb{Z}$, despite the fact that $(3,6)$ lies on the upper of the two lines mentioned in Theorem 3.1.

The example also shows that Theorem 3.3 is not true for $\overline{\mathcal{H}}^{\prime}(D)$. In this context, let us recall some facts from [Lee-2] and [Ra]: If $D$ is a knot diagram, all elements of $\overline{\mathcal{H}}^{\prime}(D) \otimes \mathbb{Q}$ have the same primary degree. For any diagram $D$, the filtration on $\overline{\mathcal{C}}^{\prime}(D)$ induces a spectral sequence whose $E_{2}$-term is $\overline{\mathcal{H}}(D)$ and which converges to $\overline{\mathcal{H}}^{\prime}(D)$. Therefore, $\operatorname{dim}_{\mathbb{Q}}\left(\overline{\mathcal{H}}^{\prime}(D) \otimes \mathbb{Q}\right) \leq \operatorname{dim}_{\mathbb{Q}}(\overline{\mathcal{H}}(D) \otimes$ $\mathbb{Q})$. If $D$ is a diagram of a $k$-component link, $\operatorname{dim}_{\mathbb{Q}}\left(\overline{\mathcal{H}}^{\prime}(D) \otimes \mathbb{Q}\right)=2^{k}$.

Remark. There is a decomposition of $\overline{\mathcal{C}}^{\prime}(D) \otimes \mathbb{Q}$ (which does not respect the filtration) into a contractible complex and a complex of dimension $2^{k}$ with trivial differential. Let us briefly describe this decomposition. Let $\{a, b\} \subset \mathcal{A} \otimes \mathbb{Q}$ be the basis of $\mathcal{A} \otimes \mathbb{Q}$ defined in [Lee-2]. We call $a$ and $b$ colors. A colored Kauffman state is a Kauffman state $D^{\prime}$ together with an assignment of $a$ or $b$ to every circle of $D^{\prime}$. By $(8)$ and $(10), \overline{\mathcal{C}}^{\prime}(D) \otimes \mathbb{Q}$ is spanned by vectors corresponding to colored Kauffman states of $D .^{7} \mathrm{~A}$ coloring of a diagram $D$ is an assignment of $a$ or $b$ to every edge of $D$. A coloring of $D$ is called admissible if for every crossing of $D$ either all four edges touching at the crossing have the same color or two neighboring edges (for example the lower left edge and the lower right edge in $\times$ ) have color $a$ and the other two edges have color $b$. If $c$ is an admissible coloring of $D$, we may smoothen every two-color crossing of $D$ in the way consistent with the coloring to obtain a colored diagram $D_{c}$ in which every component is colored consistently. Let $V\left(D_{c}\right)$ denote the subspace of $\overline{\mathcal{C}}^{\prime}(D) \otimes \mathbb{Q}$ spanned by all colored Kauffman states of $D$ whose colorings agree with the coloring of $D_{c}$. The structure of $d^{\prime}$ implies that $V\left(D_{c}\right)$ is a subcomplex of $\overline{\mathcal{C}}^{\prime}(D) \otimes \mathbb{Q}$, whence we get a decomposition of $\overline{\mathcal{C}}^{\prime}(D) \otimes \mathbb{Q}$ (which does not respect the filtration):

$$
\overline{\mathcal{C}}^{\prime}(D) \otimes \mathbb{Q}=\bigoplus_{c \text { admissible }} V\left(D_{c}\right) .
$$

The subcomplexes $V\left(D_{c}\right)$ are easy to understand: if $D_{c}$ has at least one crossing, $V\left(D_{c}\right)$ is isomorphic to the mapping cone of an isomorphism and hence conctractible. If $D_{c}$ has no crossings, $V\left(D_{c}\right)$ is one-dimensional and hence has trivial differential. The admissible colorings $c$ of $D$ for which $D_{c}$

\footnotetext{
${ }^{7}$ This description of $\overline{\mathcal{C}}^{\prime}(D) \otimes \mathbb{Q}$ is similar to the description of $\overline{\mathcal{C}}(D)$ given in [Vi].
} 
has no crossings correspond bijectively to the possible orientations of $D$ (see [Lee-2] and [Ra]). Hence there are $2^{k}$ such colorings.

\section{Acknowledgements}

The author was partially sponsored by the Swiss National Science Foundation. The author wishes to thank Norbert A'Campo and Sebastian Baader for many stimulating conversations.

\section{References}

[AP] Marta M. Asaeda, Józef H. Przytycki, Khovanov homology: torsion and thickness, arXiv:math.GT/0402402, 2004.

[BN] Dror Bar-Natan, On Khovanov's Categorification of the Jones polynomial, Algebraic and Geometric Topology 2, 2002, 337-370, arXiv:math.QA/0201043 .

[Ka-1] Louis H. Kauffman, Formal Knot Theory, Mathematical Notes 30, Princeton University Press, 1983.

[Ka-2] Louis H. Kauffman, State models and the Jones polynomial, Topology 26, 1987, 395-407.

[Kh-1] Mikhail Khovanov, A categorification of the Jones polynomial, Duke Math. J. 101, 2000, no. 3, 359-426, arXiv:math.QA/9908171, 2001.

[Kh-2] Mikhail Khovanov, A functor-valued invariant of tangles, arXiv:math.QA/0103190, 2001.

[Ko] Ilya S. Kofman, Private communication; an abstract of the talk on spanning trees and Khovanov homology at the "Knots in Washington XVIII" conference in May 2004 can be found at http://at.yorku.ca/c/a/n/v/19.htm .

[ML] Saunders Mac Lane, Homology, Die Grundlehren der mathematischen Wissenschaften Band 114, Springer, 1963.

[Lee-1] Eun Soo Lee, The support of Khovanov's invariants for alternating knots, arxiv:math.GT/0201105, 2002.

[Lee-2] Eun Soo Lee, On Khovanov invariant for alternating links, arXiv:math.GT/0210213, 2002.

[OSz] Peter Ozsváth, Zoltán Szabó, Knot Floer homology, genus bounds, and mutation, arXiv:math.GT/0303225, 2003. 
[Ra] Jacob Rasmussen, Khovanov homology and the slice genus, arXiv.math.GT/0402131, 2004.

[Sh-1] Alexander Shumakovitch, Private communication.

[Sh-2] Alexander Shumakovitch, Torsion of the Khovanov homology, arXiv:math.GT/0405474, 2004.

[Th] Morwen B. Thistlethwaite, A spanning tree expansion of the Jones polynomial, Topology 26, 1987, 297-309.

[Vi] Oleg Viro, Remarks on the definition of Khovanov Homology, arXiv:math.GT/0202199, 2002.

[We] Stephan M. Wehrli, Khovanov Homology and Conway Mutation, arXiv:math.GT/0301312, 2003.

E-mail address: wehrli@math-lab.unibas.ch 